\title{
ACOUSTIC WAVE-BASED FOREST FIRE EXTINGUISHER AND DETECTION USING MACHINE
}

\author{
Aarthi V \\ Department of Electronics and Communication \\ Engineering, \\ Arasu Engineering College, \\ Anna University, \\ Kumbakonam, Thanjavur, \\ Tamilnadu, \\ India. \\ Karthika K \\ Department of Electronics and Communication \\ Engineering, \\ Arasu Engineering College, \\ Anna University, \\ Kumbakonam, Thanjavur, \\ Tamilnadu, \\ India.
}

\begin{abstract}
Apart from causing tragic loss of lives and valuable natural and individual properties including thousands of hectares of forest and hundreds of houses, forest fires are a great menace to ecologically healthy grown forests and protection of the environment. Every year, thousands of forest fire across the globe cause disasters beyond measure and description. This issue has been research interest for many years; there are very well studied solutions available out there for testing or even ready for use resolve this problem. Currently, there are many different solutions to detect the forest fire. people are using sensors to detect the fire. But this case is not possible for large acres of forest. In this paper we discuss a new approach for fire detection, in which modern technologies are used. In particular, we propose a platform that artificial intelligence. The computer vision methods for recognition and detection of smoke and fire based on the still images or the input videos from the cameras. The accuracy is based on algorithm which we are going to use and the datasets and splitting them into train and test set. A Sonic Fire Extinguisher extinguish fire by sound waves. Generally, fire is extinguished with the help of water or carbon dioxide. Extinguishing fire through sound bass sounds like crazy.
\end{abstract}

Keywords-forest fire detection, machine learning, computer vision, sonic fire extinguisher

\author{
Abirami J \\ Department of Electronics and Communication \\ Engineering, \\ Arasu Engineering College, \\ Anna University, \\ Kumbakonam, Thanjavur, \\ Tamilnadu, \\ India \\ Kiruba R \\ Department of Electronics and Communication \\ Engineering, \\ Arasu Engineering College, \\ Anna University, \\ Kumbakonam, Thanjavur, \\ Tamilnadu, \\ India
}

\section{INTRODUCTION}

The most up to date information on the current bush fire season in Australia. According to the latest report, which they provided for 2019-20 commenced with serious uncontrolled fires in June 2019. Hundreds of fires have been or still are burning, mainly in the southeast of the country. This year of January 14, fires this season have burnt an estimated 18.6 million hectares (46 million acres; 186,000 square kilometers; 72,000 square miles) destroyed over 5,900 buildings (including 2,779 homes) and killed at least 34 people. The dramatic effects of wildfires have cause damages of over480 millions of mammals, birds and reptiles were lost since September with concerns the entire species of plants and animals may have been wiped out by bushfire, later explained to more than a billion. New south wales also experienced the longest continuously burning bushfire complex in Australia's history, having burnt more than 4 million hectares $(9,900,000$ acres $)$, with 70-metre high $(230 \mathrm{ft})$ being reported. In 2019 Amazon rainforest [2] wildfires burnt 900,000 hectares $(2,200,000$ acres) of land. In a time where environmental issues are becoming increasingly political. Some forest fire informs also that the report is showing a decrease in the number of fires, compared to the number fires, which occurred annually during the last decade. This decrease can be explained with the more severe actions and sanctions to the people that caused wildfires and with the introduction of more advanced technical solutions for forest fire detection. This reason presents the necessity to constantly 
develop implemented and upgrade the solutions and system for fire detection. The result in devastating loss and irreparable damage to the environment and atmosphere (30\% of carbon dioxide (co2) in the atmosphere comes from the forest fire). To avoid uncontrollable wide spreading of forest fires, it is necessary to detect fires in early state and to prevent the propagation. It is important to move adequate fire equipment and qualify operational manpower as fast as possible to the source of the fire. Furthermore, and adequate logistical infrastructure for sufficient supply with extinguishing devices can maintenance is necessary as well as continuous monitoring of the fire spread. Moreover, the training of personnel is an important component for successful combating of forest fires. An integrated approach for forest fire detection and suppression is based on combination of different detection system depending on wild fire risk, a size of the area and human presence, consisting of all necessary part such as early detection, remote sensing technique, logistics and training by simulation and fire fighting vehicles. The most important factors in the fight against forest fires include the earliest possible detection of the fire event, the proper categorization of the fire and fast response from the firefighting departments.

The aim proposed platform is not only to use modern technologies, but also to improve the above-mentioned factors by reducing fire detection time, by minimizing the false alarms and by issuing of timely responses and notifications to the fire services in case of real forest fires.

In this paper we discuss the proposed platform for early forest fire detection using image processing technique3[13]. Which involves cameras both optical and thermal. For very large and low risk areas satellite and aero monitoring is possible. Especially in the several hundred observation towers equipped with cameras-based systems have been setup to observe forests. Recorded still images or videos sequences are transmitted to a control Centre and analyzed by appropriate software (open cv). If a fire is clearly identified, fire suppression is initialized by an alarm going directly to the fire brigade. By keeping the user informed on the state of things and giving the users control of things, a better global humans-devices-humans communication can be achieved.

The platform is completely automated on-board computers and processing capabilities. They can detect fires based on the data captured by their thermal cameras and they can process this data without the need for centralized computing engines. In addition, and to further improve the platform, we have planned to implement artificial intelligence by allowing the cameras to make fire prediction based on the computer vision techniques. In order to implement the artificial intelligence solution, we will rely on and use machine learning[1].

Machine learning are currently very hot topic in the computing systems, because of the ability to provide algorithm, development and training toolkits, data, as well as computer power to design, train and deploy models into applications, process and automation. Currently used in a wide range of enterprise applications, mostly involving prediction or classification.

\section{CONCEPTUAL MODEL OF A PLATFORM FOR EARLY FIRE FOREST FIRE DETECTION}

This section describes the conceptual model of a platform for early forest fire detection that is going to be implemented under an international project entitled "Early Forest Fire Detection Using Drones and AI.This project was designed with the intention to create a transnational cooperation among the countries in the Australia-Mediterranean Area and more specifically between Greece and Cyprus. The goal of the project is to achieve the proof of the effectiveness of the technology and the implementation of a system for early detection and prevention of wildfires targeting a positive environmental impact and climate change resiliency. All of the three developed platforms will be part of one system under the name THEASIS. The focus of this paper is to investigated the possibilities and development stages of the platform for early forest fire detection

\section{A. Model of the platform for early forest fire detection -}

Fire detection systems for outdoor environment could be implemented by using specialized cameras, which are able to capture multispectral images. The biggest challenge that arises in these setups is where to place the camera(s) in order to have the best view on the observed territory. Since these systems have their limitations, since they provide stationary point of view, we have use observation towers equipped cameras approach.
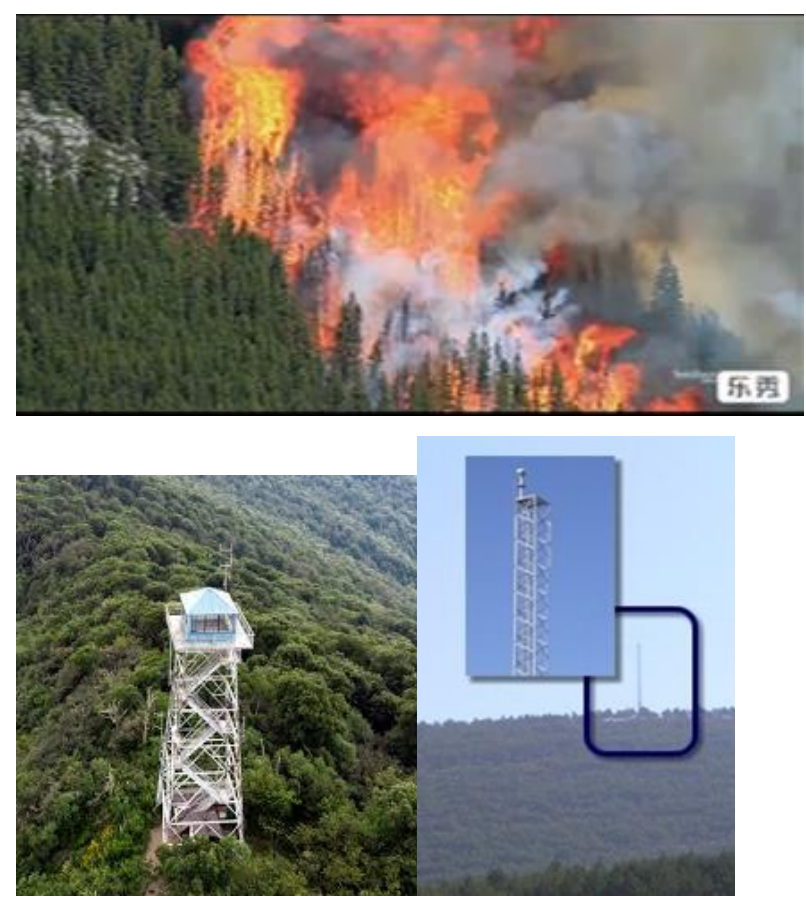

Fig.1: Platform for early forest fire detection fixed with towers and specialized cameras 


\section{International Journal of Engineering Applied Sciences and Technology, 2020 \\ Vol. 4, Issue 10, ISSN No. 2455-2143, Pages 196-202 \\ Published Online February 2020 in IJEAST (http://www.ijeast.com)}

Which are going to patrol above the desired territory and will constantly observe for fire related events. The cameras will be able to capture still images or videos. In addition to, the equipped tower connected to the base station to setup the GPS and GSM services to provide the feedback about their observations. (Fig. 1).

The observation points are located in study, protected metal towers. The usual height of these towers is 35 meters. But is possible to install towers of varying sizes

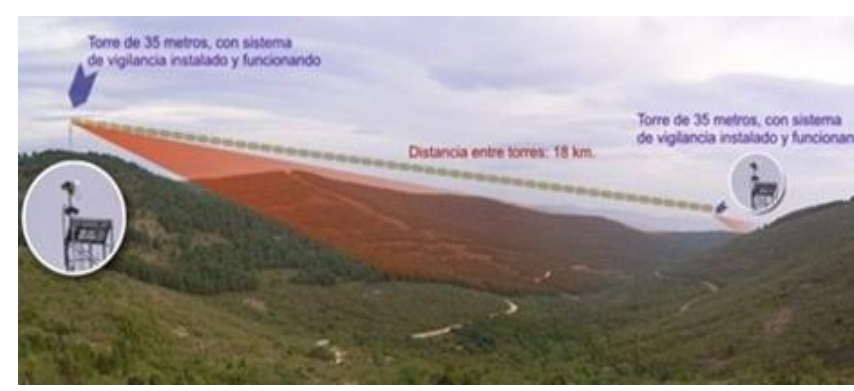

Fig.2: Fixed observation points.

These towers are equipped with an optronic system (thermal camera, CCTV camera) which equipped with functions continuously and rotates 360 degrees. As soon as the thermal cameras detect level1 alarms (source of fire) or level2 (moving objects) a signal is automatically sent via a land or by satellite to the control center (depending on the pre-established configuration in the center). Together with this notification the captured images of the alarm are also sent along to be analyzed later. In addition, meteorological data is also such as humidity, atmospheric pressure, wind speed and direction, etc.

In this block diagram(fig3.) represents the, forest fire detection process. If any fire is detected within the camera range, using the open cv[11] method to recognized the still images and videos using machine learning approach, the threshold will be checked and confirmed that fire is present. The information will be sent to nearby forest fire station through messages and alarms. The status of the fire sent to the Arduino board, to predict the value in binary data. Then the sound waves into Arduino in the range of 30-70 HZ. This range of frequency amplified using amplifier. To get the high frequency range (sound waves) to using put off the fire using sonic fire extinguisher. And also, this operation maintains by the primary station

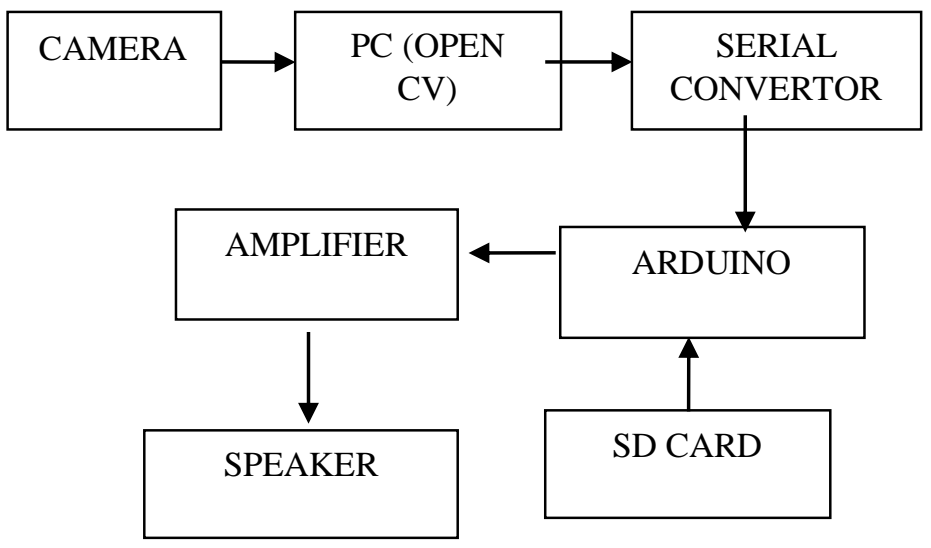

Fig.3: Block diagram of the operating principle of the forest fire detection platform

In this flow of data(fig4) through an information system, modelling its process aspects. Here the camera will capture the images and then this image undergoes filtering process, that is independent and dependent values are used. This variable splitted into $70 \%$ train set $30 \%$ of test sets. This set of variables separated into labelled data. The labelled data's fed into the knn algorithm, to checking accuracy. Get a 97\% accuracy to using algorithm clearly. The process done by the confusion matrix process. Once we get an accuracy, to signal turn on camera, this hsv, resize, blur characteristics are used and image segmentation process to be done. Finally got the output of the fire detection this above process

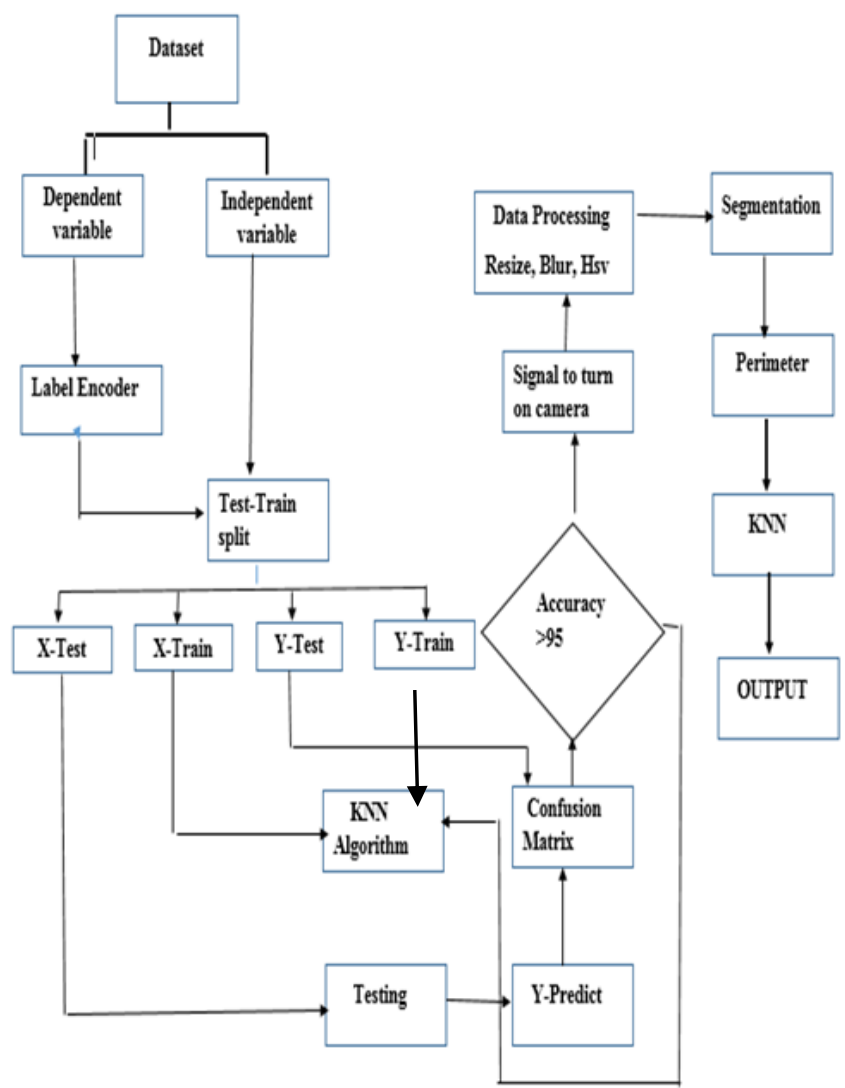

Fig.4: Flowchart of the operating principle of the forest fire detection platform

In this software using python to detect the accuracy using $\mathrm{knn}$ algorithm. This algorithm to find out $\mathrm{k}$ nearest neighbour value. This value found using Euclidian distance.

Euclidian distance $=\sqrt{(a-b)^{2}+(y-b)^{2}}$ 


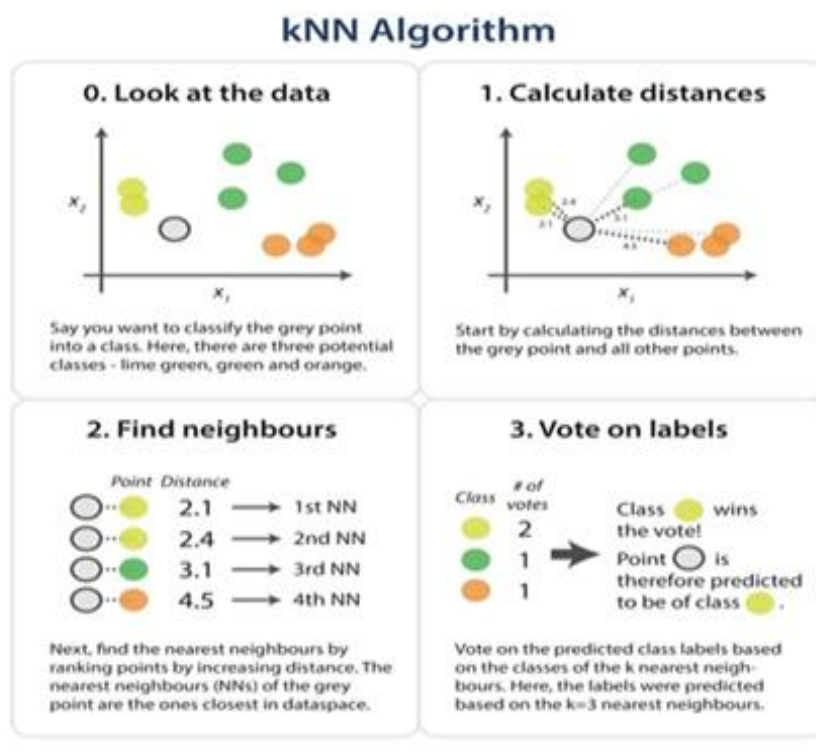

Fig.5: KNN Algorithm

The k-Nearest neighbours algorithm or knn for short is a very simple technique. The entire training dataset is stored. when a prediction is required, the k-most similar records to a new record from the training dataset are then located. From this neighbour a summarized prediction is made. Similarity between records can be measured many different ways. A problem is data specific method can be used. Generally, with data, a good starting point is the Euclidian distance.

Once the neighbor is discovered, the summary prediction can be made by returning the most common outcome are taking the average. As such, knn can be used for classification or regression problems. There is no model to speak of other then holding the entire training dataset. Because no work is done until a prediction is required, knn is often refer to as a lazy learning method. In this machine learning, the knn algorithm classification is done by a majority votes to its neighbors

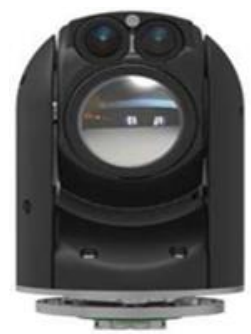

Fig.6: The NightHawk2 camera and its parameters [12]

a. Zoom: x20 + x2 digital (total x40),

b. HFOV: 60o WFOV - 3oWFOV - 1.5Odfov, c. Thermal Resolution: 640x480,

d. Pitch FOR: -450 to +1350 ,

e. Roll FOR: -180 o to +180 o,

f. Weight: 250grams,

g. Dimensions: Diameter $=64 \mathrm{~mm}$, Height $=94 \mathrm{~mm}$.

(fig6) The telemetry and control links between the tower and its ground station are completed using two channels for data communication. One of the channels is duplex for simultaneous bidirectional control and data transfer, while the other is used as radio channel for high definition real time video streaming. The second channel is set to work in simplex mode for downstream video transfer. That video is going to be used for analysis of the observed area .The high-altitude tower will be equipped with Nighthawk 2 EO/IR camera with 20x zoom and thermal resolution of $640 \times 480$ as shown in Fig4. This camera is able to capture different temperature levels, and once the UAV detects increased or abnormal temperature levels it will immediately raise an alarm and will send the GPS coordinates of the problematic area to its base station. The camera weighs only 250 grams, which will not cause significant downgrade in the drone performance and will not reduce its endurance

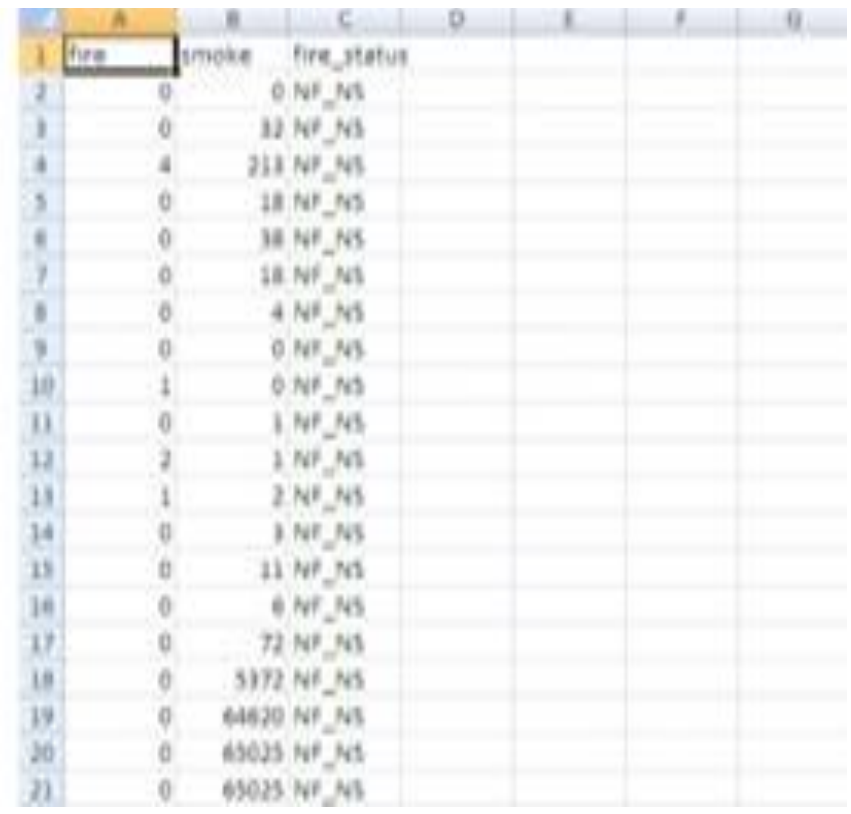

Fig.7: CSV (Comma delimited) file.

\section{B. Development stages -}

There are three main development stages for bringing the platform for early forest detection in action. They include the planning, the designing and the building of the system. In the implementation process, most of the project team will be involved with distributed tasks. The planning stage involves 
meetings of the team, discussions and building of a conceptual model, as well as defining the specifications of the equipment that is going to be purchased. The designing stage involves Pre building tasks, which include clarification of the conceptual model, purchasing of the equipment and additional discussion and talks. The last and most difficult stage from the development process is the actual building of the system. This stage involves actions that are separated in two main tasks: preparation of the system components and laboratory testing of components. These actions must be implemented before the actual use of the system. The preparation of the system components involves tasks as components testing, assembling of the components, camera testing, DSP module testing, GPS module tests and also troubleshooting of the system, while the laboratory testing involves practical tests of the UAVs and their components and laboratory testing of the fire detection system.

In addition, the system could be improved by implementing computer vision techniques. For this purpose, we have used artificial intelligence concepts for training a machine learning to recognize smoke in images taken from the cameras. Before providing more details about our CV implementation, we have included some basic information about artificial intelligence, machine learning, deep learning and the connection between them. smoke and fire detection using images can be defined as binary classification problem, in which we have as input an image, which has to be classified as containing smoke and fire or not. If it is classified as image, which contains smoke, the reported output value is one. Otherwise, the output value is zero of the paper will be sent. Proofs are sent to the corresponding author only.

\section{ARTIFICIAINTELLIGENCE,MACHINE LEARNING, IMAGE DESCRYPTION}

Artificial Intelligence has become extremely popular in the recent years as it has the ability to perform task, which are inherent to a human mind. Artificial intelligence, sometime referred to as machine intelligence is implemented by using machine learning.

The machine learning [9] are specialized computer models, which can be trained to perform different tasks. They are used for classification of image segmentation, counting pixels. There are several algorithms are used in machine learning, but the most widely used for image detection and computer vision methods. Depending on the datasets, we have machine learning methods are used. In terms of methods for training and testing the datasets References

Detection accuracy can be improved by using machine learning techniques. Machine learning is an effective approach to study the correlation that exists between the output and the input features considered. There are four steps for machine learning procedure. 1) Feature selection and output labeling, 2) Sample collection 3) Offline training 4) Online classification. [2]

Feature selection[10] is the method in which most significant parameter is chosen, which will help to describe the problem easily. The parameters are chosen in such a way that maximum variability in the model is explained by them. Outputs are classified according to the knowledge of the expects in output labeling method. Second is the sample collection in which data is gathered for training and back end system is kept as a database of collection of data. It is also called centralized learning. However, in many real-life scenarios is not applicable. So decentralized or distributed learning is preferred. Third step is offline training in which classification is done between two or more solutions and they do not have resource constrain. Lastly the online classification is different from offline because offline is done by keeping a backend server but in online only learning overhead is considered.

First, the image run the track bar program and get the results like this snapshot. We will get the track bar and we can adjust to any color value to segment using hsv values.second, image adjusting the track bar and select some specific color which we want, then segment the color using hsv upper and lower limit

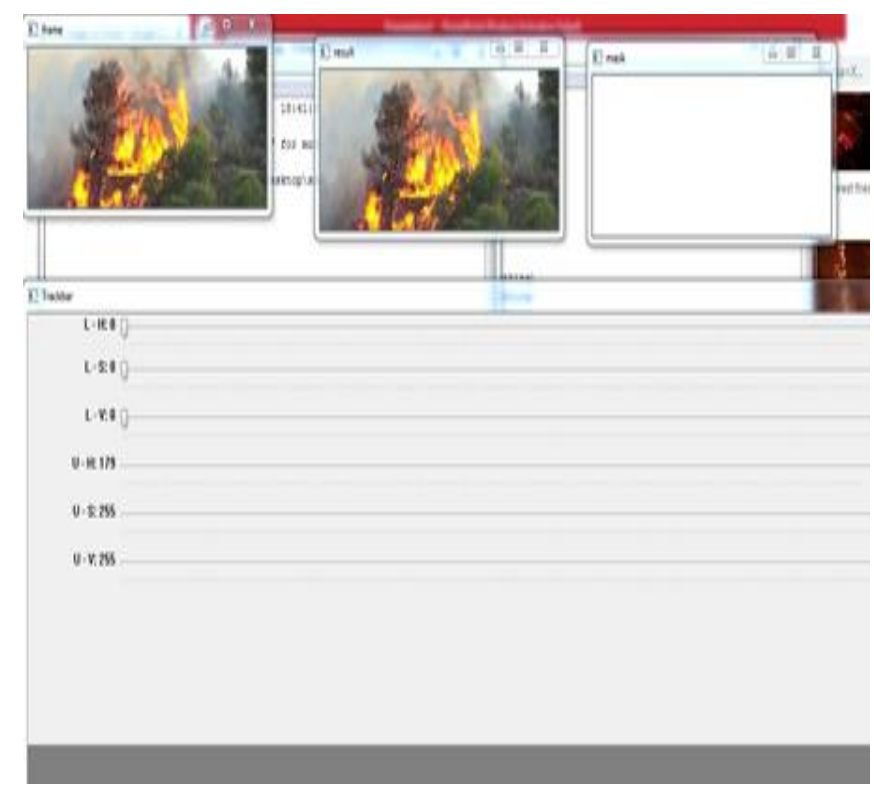

Fig.8 : Trackbar

In this figure trackbar is used to extract the expected colour area from the image. Which shoes the colour and three trackbar to specify each of B, G, R colours. By default initial colour set to be black.For cv2.getTrackbarPos() function, first argument is the trackbar name, second one is the window name to which it is attached, third argument is the default value, fourth one is the maximum value and fifth one is the callback function which is executed every time trackbar value changes. The callback function always has a default argument which is the trackbar position 


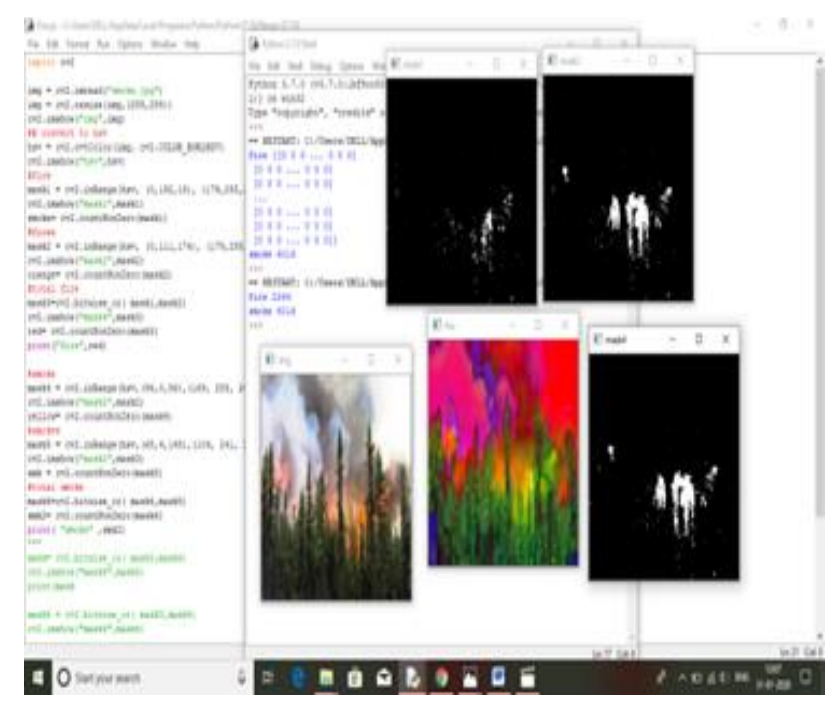

Fig.9 : Image segmentation.

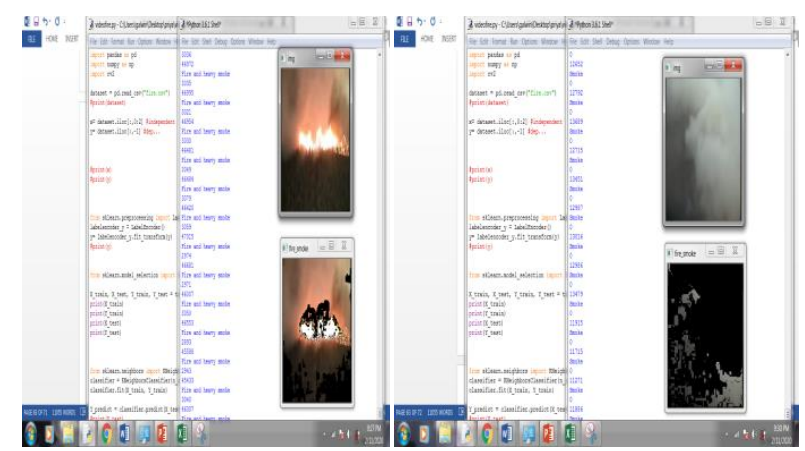

Fig.10: Fire detection and Smoke detection

Using the hsv value we predicted from the trackbar program, segmented the selected color. Using non zero count method predicting the pixel values from the image we segmented.

\section{CONCLUSION}

The system for early forest fire detection is still in its development stage. We are still waiting for some equipment to be purchased, but we have planned and discussed the actual implementation. We have performed a thorough research and some simulation experiments and we believe that we follow the right way to achieve the goal. We also believe that we apply adequate approach that is also up-to-date. We think that the system could enhance the available platforms for fire detection and we hope that such improvement could significantly reduce the damages caused by untimely or late fire detection

\section{REFERENCE}

[1] Diyana Kinaneva, Georgi Hristov, Jordan Raychev and Plamen Zahariev (2019) "Early Forest Fire Detection Using Drones and Artificial Intelligence" MIPRO 2019, May20-24, 2019, Opatija Croatia DOI No: 10.23919/MIPRO.2019.8756696

[2] Md. Saif Allauddin, G. Sai Kiran, GSS. Raj Kiran, G Srinivas Uma Ratna Mouli, P Vishnu Prasad (2019)" Development of a Surveillance System for Forest Fire Detection and Monitoring using Drones" 978-1-53869154-0/19/\$31.00 C2019 IEEE IGARSS 2019 DOI No: 10.1109/IGARSS.2019.8900436

[3] Thou-Ho (Chao-Ho) Chen, Yen-Hui Yin, Shi-Feng Huang and Yan-Ting Ye(2006)'The Smoke Detection for Early Fire-Alarming System Base on Video Processing" International Conference on Intelligent Information Hiding and Multimedia Signal Processing (IIH-MSP'06)0-7695$2745-0 / 06 \$ 20.00$ C 2006 DOI No: $10.1109 / \mathrm{IIH}-$ MSP.2006.265033

[4] C. EmmyPrema1 and S. S. Vinsley (2014)" Image Processing Based Forest Fire Detection using $\mathrm{YCbCr}$ Colour Model"-2014 doi-10.1109/iccpct.2014.7054883

[5] Benamar Kadri, Benamar Bouyeddou and Djillali Moussaoui (2018) "Early Fire Detection System Using Wireless Sensor Networks "DOI 10.1109/icass.2018.8651977

[6] A. Kansal, Y. Singh, N. Kumar and V. Mohindru, (2015) "Detection of forest fires using machine learning technique: A perspective," 2015 Third International Conference on Image Information Processing (ICIIP), Waknaghat, 2015, pp. 241-245. doi: 10.1109/ICIIP.2015.7414773

[7] M. S. Allauddin, G. S. Kiran, G. R. Kiran, G. Srinivas, G. U. R. Mouli and P. V. Prasad, "Development of a Surveillance System for Forest Fire Detection and Monitoring using Drones," IGARSS 2019 - 2019 IEEE International Geoscience and Remote Sensing Symposium, Yokohama, Japan, 2019, pp. 9361-9363.doi: 10.1109/IGARSS.2019.8900436

[8] M. D. Molovtsev and I. S. Sineva, "Classification Algorithms Analysis in the Forest Fire Detection Problem," 2019 International Conference "Quality Management, Transport and Information Security, Information Technologies" (IT\&QM\&IS), Sochi, Russia, 2019, pp. 548-553.doi: 10.1109/ITQMIS.2019.8928398

[9] L. Zhang, L. Mu, Z. Liu, Y. Zhang and J. Ai, "Automated Maneuvering Decision for UAVs in Forest Surveillance and Fire Detection Missions*," 2018 International Conference on Unmanned Aircraft Systems (ICUAS), Dallas, TX, 2018, pp. 1085-1090.doi: 10.1109/ICUAS.2018.8453322

[10] Guangyi Wang, Youmin Zhang, Yaohong Qu, Yanhong Chen (2019)"Early Forest fire detection fire region 
segmentation based on deep learning" The 31th Chinese Control and Decision Conference (2019 CCDC) DOI 10.1109/CCDC.2019.8833125

[11]B. Ugur Toreyin, A. Enis Cetin (2008) "Computer Vision Based Forest Fire Detection" 978-1-4244-19999/08/\$25.00@20081EEE

DOI 10.1109/SIU.2008.4632677

[12]M. D. Molovtsev and I. S. Sineva, "Classification Algorithms Analysis in the Forest Fire Detection Problem," 2019 International Conference "Quality Management, Transport and Information Security, Information Technologies" (IT\&QM\&IS), Sochi, Russia, 2019, pp. 548-553.doi: 10.1109/ITQMIS.2019.8928398

[13] O. Elsarrar, M. Darrah and R. Devine, "Analysis of Forest Fire Data Using Neural Network Rule Extraction with Human Understandable Rules," 2019 18th IEEE International Conference On Machine Learning And Applications (ICMLA), Boca Raton, FL, USA, 2019, pp. 1917-19176.doi: 10.1109/ICMLA.2019.00308

[14]D. Kinaneva, G. Hristov, J. Raychev and P. Zahariev, "Application of Artificial Intelligence in UAV platforms for Early Forest Fire Detection," 2019 27th National Conference with International Participation (TELECOM), Sofia, Bulgaria, 2019, pp. 50-53.doi: 10.1109/TELECOM48729.2019.8994888

[15]H. Li, X. Fei and C. He, "Study on Most Important Factor and Most Vulnerable Location for a Forest Fire Case Using Various Machine Learning Techniques," 2018 Sixth International Conference on Advanced Cloud and Big Data (CBD), Lanzhou, 2018, pp. 298-303.doi: 10.1109/CBD.2018.00060

[16] R. Kaabi, M. Sayadi, M. Bouchouicha, F. Fnaiech, E. Moreau and J. M. Ginoux, "Early smoke detection of forest wildfire video using deep belief network," 2018 4th International Conference on Advanced Technologies for Signal and Image Processing (ATSIP), Sousse, 2018, pp. 1-6.doi: 10.1109/ATSIP.2018.8364446

[17]N. Ghassempour, J. J. Zou and Y. He, "A SIFT-Based Forest Fire Detection Framework Using Static Images," 2018 12th International Conference on Signal Processing and Communication Systems (ICSPCS), Cairns, Australia, 2018, pp. 1-7.doi: 10.1109/ICSPCS.2018.8631711

[18] M. Anshori, F. Mar'i, M. W. Alauddin and W. F. Mahmudy, "Prediction of Forest Fire using Neural Network based on Extreme Learning Machines (ELM)," 2019 International Conference on Sustainable Information Engineering and Technology (SIET), Lombok, Indonesia, 2019, pp. 301-305.doi: 10.1109/SIET48054.2019.8986106

[19] S. Wu and L. Zhang, "Using Popular Object Detection Methods for Real Time Forest Fire Detection," 2018 11th International Symposium on Computational Intelligence and Design (ISCID), Hangzhou, China, 2018, pp. 280284.doi: 10.1109/ISCID.2018.00070

[20] Yanhong Chen, youmin Zhang, Jing xin, yingmin Yi(2018) "A UAV-based Forest Fire Detection Algorithm Using
Convolutional Neural Network" Proceedings of the 37th Chinese Control Conference

July25-27, 2018, Wuhan, China DOI10.23919/chiCC.2018.8484035 